\title{
AVALIAÇÃO DA ATENÇÃO PRIMÁRIA: COMPARATIVO ENTRE O DESEMPENHO GLOBAL E AS AÇÕES DE HANSENÍASE
}

\section{EVALUATION OF PRIMARY ATTENTION: A COMPARATIVE BETWEEN GLOBAL PERFORMANCE AND LEPROSY ACTIONS}

\section{EVALUACIÓN DE LA ATENCIÓN PRIMARIA: COMPARATIVO ENTRE EL DESEMPEÑO GLOBAL Y LAS ACCIONES DE LA LEPRA}

\author{
Nayara Figueiredo Vieira ${ }^{1}$, Rayssa Nogueira Rodrigues ${ }^{2}$, Eyleen Nabyla Alvarenga Niitsuma ${ }^{3}$, Fernanda Moura Lanza ${ }^{3}$, Francisco \\ Carlos Félix Lana ${ }^{5}$
}

\begin{abstract}
RESUMO
Objetivo: comparar a presença e extensão dos atributos da atenção primária no geral e no desempenho da hanseníase. Métodos: estudo transversal e avaliativo, realizado em um município de Minas Gerais, com 28 profissionais da Estratégia Saúde da Família, sendo composto por médicos e enfermeiros. Para a coleta de dados foram aplicados o Primary Care Assessment Tool e o Instrumento de Avaliação de Desempenho da Atenção Primária nas Ações de Controle da Hanseníase. Utilizou-se os testes estatísticos Wilcoxon e $t$ de Student com significância $p<0,05$. Resultados: A atenção primária, no global, apresentou alta orientação no escore geral e derivado. Nas ações de hanseníase, apenas o escore essencial alcançou alta orientação. Encontrou-se diferença significativa no escore essencial, atributos acesso e integralidade dos serviços disponíveis, e no escore derivado, atributo orientação comunitária. Conclusão: o desempenho da atenção primária no geral é melhor, o que demonstra fragilidades na integração das ações de controle da hanseníase e necessidade de fortalecimento das estratégias de enfrentamento da endemia no que concerne à Estratégia de Saúde da Família.
\end{abstract}

Descritores: Pesquisa sobre Serviços de Saúde; Atenção Primária à Saúde; Hanseníase; Enfermagem.

\begin{abstract}
Objective: to compare the presence and extent of primary care attributes in general and in leprosy performance. Methods: crosssectional and evaluative study, carried out in a city of Minas Gerais, with 28 professionals from the Family Health Strategy, consisting of doctors and nurses. The Primary Care Assessment Tool and the Primary Care Performance Assessment Instrument for Leprosy Control Actions were applied for data collection. Wilcoxon statistical test and Student's t test were used with $p<0.05$. Results: Primary care, overall, showed high orientation in the general and derived score. In leprosy actions, only the essential score reached high orientation. There was significant difference in the essential score, access and integrality attributes of the available services, and in the derived score, attribute community orientation. Conclusion: the primary care general performance is better, which demonstrates weaknesses in the integration of leprosy control actions and the need to strengthen the endemic coping strategies in relation to the Family Health Strategy.
\end{abstract}

Descriptors: Health Services Research; Primary Health Care; Leprosy; Nursing.

\section{RESUMEN}

Objetivo: comparar la presencia y extensión de los atributos de la atención primaria en general y en el desempeño de la lepra. Métodos: estudio transversal y evaluativo, realizado en un municipio de Minas Gerais, con 28 profesionales de la Estrategia Salud de la Familia, compuesto por médicos y enfermeros. Para la recolección de datos, se aplicaron el Primary Care Assessment Tool y el Instrumento de Evaluación de Desempeño de la Atención Primaria en las Acciones de Control de la Lepra. Se utilizaron las pruebas estadísticas de Wilcoxon y $\mathrm{t}$ de Student con significancia $\mathrm{p}<0,05$. Resultados: La atención primaria, en general, presentó alta orientación en el puntaje general y derivado. En las acciones de lepra, sólo el puntaje esencial alcanzó alta orientación. Se encontró diferencia significativa en el score esencial, atributos acceso e integralidad de los servicios disponibles, y en el puntaje derivado, atributo orientación comunitaria. Conclusión: el desempeño de la atención primaria en general es mejor, lo que demuestra fragilidades en la integración de las acciones de control de la lepra y necesidad de fortalecer las estrategias de enfrentamiento de la endemia en lo que concierne a la Estrategia de Salud de la Familia.

Descriptores: Investigación en Servicios de Salud; Atención Primaria de Salud; Lepra; Enfermería.

${ }^{1}$ Graduada em Enfermagem. Doutoranda em Enfermagem pela Universidade Federal de Minas Gerais. Professora da Faculdade de Ciência da Saúde de Unaí. ${ }^{2}$ Graduada em Enfermagem. Doutoranda em Enfermagem pela Universidade Federal de Minas Gerais. Professora Substituta da Universidade Federal de São João del Rei. ${ }^{3}$ Graduada em Enfermagem. Doutoranda em Enfermagem pela Universidade Federal de Minas Gerais. Docente no Instituto Federal de Educação do Norte de Minas Gerais. ${ }^{4}$ Graduada em Enfermagem. Doutora em Enfermagem pela Universidade Federal de Minas Gerais. Docente na Universidade Federal de São João del-Rei. ${ }^{5}$ Graduado em Enfermagem. Doutor em Enfermagem Interunidades pela Universidade de São Paulo/Ribeirão Preto. Docente na Universidade Federal de Minas Gerais.

Como citar este artigo:

Vieira NF, Lana FCF, Rodrigues RN, et al. Avaliação da Atenção Primária: Comparativo Entre o Desempenho Global e as Ações de Hanseníase. Revista de Enfermagem do Centro Oeste Mineiro. 2019;9:e2896. [Access http://dx.doi.org/10.19175/recom.v9i0.2896 ; Available in: DOI: 


\section{INTRODUÇÃO}

Os serviços de Atenção Primária à Saúde (APS) são porta preferencial de entrada do usuário no Sistema Único de Saúde (SUS) e nela são oferecidas, por equipe multiprofissional, ações de promoção da saúde, prevenção de agravos, proteção, diagnóstico, tratamento, reabilitação, redução de danos, cuidados paliativos e vigilância em saúde ${ }^{(1)}$.

Para a realização dessas ações de saúde, os serviços de APS, que no Brasil, têm a Estratégia Saúde da Família (ESF) como prioritária para a consolidação desse ponto de atenção na rede ${ }^{(1)}$, precisam implementar no cotidiano de trabalho os elementos estruturantes da APS ${ }^{(2)}$. Os elementos estruturantes são os atributos, divididos em: atributos essenciais - atenção ao primeiro contato (porta de entrada/acesso), longitudinalidade/ atendimento continuado, integralidade e coordenação - e os derivados orientação familiar, comunitária e competência cultural $^{(2)}$

Estudos que avaliaram as características estruturais da APS em municípios brasileiros, sob a ótica dos profissionais, demonstraram fraca orientação no acesso de primeiro contato ${ }^{(3-5)}$ e na longitudinalidade ${ }^{(3)}$. No entanto, a expansão da ESF permitiu avanços no acesso aos serviços de saúde e aumento da qualidade assistencial, e consequentemente, refletiu de forma positiva nos indicadores de saúde.

Dentre as doenças infectoparasitárias, a hanseníase ainda permanece como desafio na saúde pública com 26.395 casos novos em 2015, destes $67,9 \%$ nas formas clínicas multibacilares, $6,6 \%$ com grau 2 de incapacidade física e 7,3\% em menores de 15 anos $^{(6)}$. A magnitude e transcendência do agravo bem como o comportamento epidemiológico e operacional permitiram a indicação da eliminação da hanseníase como uma área estratégica da APS ${ }^{(7-8)}$, nos quais as ações devem ser desenvolvidas pelos municípios por meio do princípio da integralidade $^{(1)}$ e das diretrizes da descentralização e regionalização da assistência à saúde; cuidado centrado na pessoa; longitudinalidade e resolutividade ${ }^{(1,8)}$.

Frente à necessidade de fortalecimento das ações de controle da hanseníase (ACH), o Ministério da Saúde reforça a recomendação que a APS ofereça uma atenção continuada e integral para casos, contatos e famílias, coordenando a rede, de acordo com as necessidades, realizando ações de mobilização para favorecer o diagnóstico e redução do estigma ${ }^{(7)}$. Pesquisa que avaliou a presença e extensão dos atributos da APS na realização das $\mathrm{ACH}$ evidenciou fraca orientação da APS no acesso e orientação comunitária ${ }^{(9)}$.

Avaliar o desempenho da APS na realização das $\mathrm{ACH}$ significa identificar fragilidades $e$ potencialidades do primeiro ponto de atenção do SUS. Nesse contexto, a ferramenta Primary Care Assessment Tool (PCATool-Brasil) ${ }^{(10)}$ proporciona a avaliação dos atributos da APS e o "Instrumento de Avaliação de Desempenho da Atenção Primária à Saúde nas Ações de Controle da Hanseníase (PCAThanseníase) ${ }^{\prime(9)}$ permite avaliar o grau de orientação da APS na realização das $\mathrm{ACH}^{(9)}$. Ambos instrumentos de avaliação possuem o mesmo marco teórico ${ }^{(2)}$ e equivalência na metodologia de análise.

Torna-se oportuno questionar: como é o comportamento dos atributos da APS ao avaliar os serviços no geral e na realização das $\mathrm{ACH}$ ? $\mathrm{O}$ objetivo do presente estudo foi comparar a presença e a extensão dos atributos da APS no geral e no desempenho das $\mathrm{ACH}$.

\section{MÉTODOS}

Trata-se de uma pesquisa avaliativa com delineamento transversal, realizada no município de Lagoa Santa, situado na Região Metropolitana de Belo Horizonte/Minas Gerais, no período de fevereiro a março de 2014. O município, em 2014, apresentou taxa de detecção 5,11 casos novos/ 100 mil habitantes, que caracteriza como média endemicidade, dentre os indicadores operacionais, exame de contatos e cura (bom) $)^{(11)}$.

O cenário de estudo foi constituído por todos os serviços de APS, uma vez que na época da coleta de dados, o município dispunha de 17 ESF. Foram convidados a participarem do estudo todos os médicos e enfermeiros que atuavam nessas equipes.

Utilizou-se o PCATool-Brasil e o PCAThanseníase, ambos nas versões médicos/enfermeiros, pois permitem avaliar a presença e a extensão dos atributos da $\operatorname{APS}^{(9-10)}$. O PCATool-Brasil baseia-se nos atributos essenciais (acesso de primeiro contato - acessibilidade, longitudinalidade, coordenação, integralidade dos serviços prestados e disponíveis) e derivados (orientação familiar e comunitária) ${ }^{(10)}$. O PCAThanseníase constitui-se por atributos essenciais (porta de entrada, acesso, coordenação, atendimento continuado, integralidade dos 
serviços prestados e disponíveis) e derivados (orientação familiar, comunitária e profissional) ${ }^{(9)}$.

Os instrumentos foram aplicados por duas pesquisadoras treinadas e mediante entrevista previamente agendada, realizadas individualmente no ambiente de trabalho, após assinatura do Termo de Consentimento Livre e Esclarecido. Os participantes responderam primeiramente ao PCATool-Brasil e, após 30 dias, ao PCAT-hanseníase. As respostas dos dois instrumentos foram concedidas por meio da escala de Likert $^{(9-10)}$.

O cálculo do escore de cada atributo foi baseado na média, soma dos valores de resposta nos itens que compõe cada atributo dividida pelo número de itens. Os entrevistados que tiveram $50 \%$ ou mais de respostas "9" ("não sei/não lembro") não tiveram seus escores calculados. Atributos com menos de $50 \%$ de respostas " 9 " tiveram esses valores convertidos para " 2 " ("provavelmente não") de modo a pontuar de forma negativa as características do serviço não conhecidas $^{(12)}$. Posteriormente, transformou-se numa escala de 0 a 10 por meio da fórmula:

$$
\frac{[\text { escore obtido }-1 \text { (valor mínimo })] \times 10}{4 \text { (valor máximo })-1 \text { (valor mínimo })} .
$$

Os escores essencial e derivado foram obtidos pela média dos seus respectivos componentes. Calculou-se o escore geral que é a soma do escore médio dos componentes dos atributos essenciais acrescida aos derivados e dividida pelo número de itens dos atributos. Têmse como resultado o escore de cada atributo e os escores essencial, derivado e geral que caracterizam o grau de orientação da APS e o grau de orientação para $\mathrm{ACH}$. Assumiu-se uma APS fortemente orientada quando a média do escore fosse $\geq 6,6^{(10)}$.

Analisaram-se os dados pelo Software Statistical Package for the Social Sciences (SPSS) (versão 19) por meio do teste de Wilcoxon para as variáveis não-paramétricas e o teste $t$ Student pareado para variáveis com distribuição normal, com nível de significância $(p<0,05)$. A pesquisa atende às normas dispostas na Resolução 466/2012 do Conselho Nacional de Saúde e foi aprovada no Comitê de Ética em Pesquisa sob CAAE 24578213.2.0000.5149 e financiada pela Secretaria de Vigilância em Saúde (MS) - Edital 197/2012 e Fundação de Amparo à Pesquisa do Estado de Minas Gerais - Edital 03/2014.

\section{RESULTADOS E DISCUSSÃO}

A Tabela 1 mostra a caracterização dos participantes $(n=28)$. Destaca-se que $100 \%$ dos enfermeiros $(n=17)$ aceitaram participar da pesquisa e, em relação aos médicos, houve perda de três participantes devido às dificuldades de agendamento da entrevista e três equipes da ESF que estavam com desfalque deste profissional.

Tabela 1 - Descrição dos participantes do estudo segundo a categoria profissional. Lagoa Santa, MG, Brasil, 2014.

\begin{tabular}{|c|c|c|c|c|}
\hline \multirow{2}{*}{ Variáveis } & \multicolumn{2}{|c|}{ Médico } & \multicolumn{2}{|c|}{ Enfermeiro } \\
\hline & $\mathrm{n}$ & $\%$ & $\mathrm{~N}$ & $\%$ \\
\hline \multicolumn{5}{|l|}{ Pós-Graduação } \\
\hline Sim & 2 & 18,2 & 8 & 47,1 \\
\hline Não & 9 & 81,8 & 9 & 52,9 \\
\hline \multicolumn{5}{|c|}{ Tempo que trabalha na unidade } \\
\hline$<12$ meses & 3 & 27,3 & 9 & 52,9 \\
\hline Entre 12 a 36 meses & 6 & 54,5 & 7 & 41,2 \\
\hline$>36$ meses & 2 & 18,2 & 1 & 5,9 \\
\hline \multicolumn{5}{|c|}{ Tempo que trabalha na atenção primária à saúde } \\
\hline$<12$ meses & 2 & 18,2 & 4 & 23,5 \\
\hline Entre 12 a 36 meses & 4 & 36,4 & 6 & 35,3 \\
\hline$>36$ meses & 5 & 45,5 & 7 & 41,2 \\
\hline \multicolumn{5}{|c|}{ Número de treinamentos em ações de controle da hanseníase } \\
\hline Zero & 7 & 63,6 & 11 & 64,7 \\
\hline Um & 2 & 18,2 & 5 & 29,4 \\
\hline$>$ Dois & 2 & 18,2 & 1 & 5,9 \\
\hline \multicolumn{5}{|c|}{ Atuação em ações de controle da hanseníase } \\
\hline Sim & 4 & 36,4 & 7 & 41,2 \\
\hline Não & 7 & 63,6 & 10 & 58,8 \\
\hline \multicolumn{5}{|c|}{ Atendeu caso de hanseníase } \\
\hline Sim & 1 & 9,1 & 2 & 11,8 \\
\hline Não & 10 & 90,9 & 15 & 88,2 \\
\hline
\end{tabular}

Fonte: Elaborada pelos autores com base nas entrevistas realizadas. 
Evidencia-se que o reconhecimento da APS como porta de entrada não significa que as ações são efetivamente realizadas; $60,7 \%$ dos entrevistados relataram não realizar $\mathrm{ACH}$ e 89,3\% não atenderam casos. Acredita-se que essas condições seriam encaminhadas para o serviço especializado de média complexidade; barreiras no acesso são determinantes para utilização da
APS como porta de entrada e implicam na escolha do primeiro local de atendimento ${ }^{(9)}$.

Ao analisar a Tabela 2, em relação aos atributos essenciais, verifica-se que a APS de Lagoa Santa apresenta alta orientação $(\geq 6,6)$ dos serviços tanto na APS no geral quanto no desempenho das $\mathrm{ACH}$ nos atributos coordenação e integralidade dos serviços prestados.

Tabela 2- Comparação da presença e extensão dos atributos da atenção primária no geral e no desempenho da hanseníase na perspectiva de médicos e enfermeiros. Lagoa Santa, MG, Brasil, 2014.

PCATool-Brasil

PCAT-hanseníase

\begin{tabular}{|c|c|c|c|c|c|c|c|c|}
\hline Variáveis & 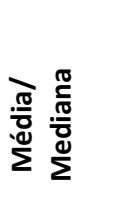 & $\begin{array}{l}\text { Desvio Padrão/ } \\
\text { Amplitude } \\
\text { interquartil }\end{array}$ & $\begin{array}{l}\% \text { Alto } \\
\text { Escore } \\
(\geq 6.6)\end{array}$ & 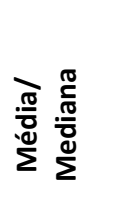 & $\begin{array}{l}\text { Desvio Padrão/ } \\
\text { Amplitude } \\
\text { interquartil }\end{array}$ & $\begin{array}{c}\text { \%Alto } \\
\text { Escore } \\
(\geq 6.6)\end{array}$ & 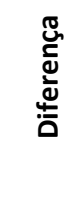 & $\frac{0}{\frac{0}{\pi}}$ \\
\hline Porta de entrada & + & + & + & $7,6 / 7,5$ & $\pm 1,4 / 0,8$ & 78,6 & + & + \\
\hline Acessibilidade/ Acesso & $3,8 / 3,7$ & $\pm 1,0 / 1,4$ & 3,6 & $5,7 / 5,5$ & $\pm 1,3 / 1,5$ & 10,7 & 1,9 & $0,001^{a}$ \\
\hline Longitudinalidade & $6,9 / 6,9$ & $\pm 1,1 / 1,2$ & 67,9 & $*$ & $*$ & $*$ & $\dagger$ & $\dagger$ \\
\hline Coordenação & $7,8 / 7,8$ & $\pm 1,0 / 0,7$ & 60,7 & $7,2 / 8,3$ & $\pm 4,9 / 7,9$ & $\ddagger$ & $-0,5$ & $0,56^{b}$ \\
\hline $\begin{array}{l}\text { Integralidade: serviços } \\
\text { prestados }\end{array}$ & $7,6 / 7,7$ & $\pm 1,1 / 1,5$ & 82,1 & $7,3 / 7,5$ & $\pm 2,1 / 3,3$ & 64,3 & $-0,2$ & $0,05^{b}$ \\
\hline $\begin{array}{l}\text { Integralidade: serviços } \\
\text { disponíveis }\end{array}$ & $6,6 / 6,6$ & $\pm 1,6 / 2,5$ & 50 & $8,9 / 8,9$ & $\pm 0,7 / 1,0$ & 100 & 2,3 & $0,001^{a}$ \\
\hline Orientação familiar & $8,6 / 8,9$ & $\pm 1,3 / 2,2$ & 96,4 & $*$ & $*$ & $*$ & $\dagger$ & $\dagger$ \\
\hline Orientação comunitária & $6,5 / 6,7$ & $\pm 1,4 / 2,1$ & 53,6 & $3,5 / 4,0$ & $\pm 2,3 / 2,7$ & 10,7 & -3 & $0,001^{a}$ \\
\hline Orientação profissional & $\dagger$ & $\dagger$ & $\dagger$ & $4,3 / 4,4$ & $\pm 2,2 / 3,1$ & 25 & $\dagger$ & + \\
\hline Essencial & $6,5 / 6,7$ & $\pm 0,8 / 1,2$ & 75 & $7,2 / 7,6$ & $\pm 1,4 / 2,6$ & 67,9 & 0,9 & $0,005^{b}$ \\
\hline Derivado & $7,6 / 7,8$ & $\pm 1,2 / 2,4$ & 67,9 & $3,9 / 4,0$ & $\pm 1,8 / 3,1$ & 10,7 & $-3,8$ & $0,001^{b}$ \\
\hline Geral & $6,8 / 6,9$ & $\pm 0,9 / 1,4$ & 75 & $6,1 / 6,3$ & $\pm 1,2 / 2,2$ & 53,6 & $-0,7$ & $0,08^{a}$ \\
\hline
\end{tabular}

Fonte: Elaborada pelos autores com base nas entrevistas realizadas.

a) Significância estatística pelo Teste $t$ Student para amostras pareadas; b) Significância estatística pelo teste de Wilcoxon para amostras pareadas.

*Não foi possível calcular os escores dos atributos atendimento continuado e orientação familiar, dimensões que são respondidas por profissionais que atenderam casos da doença, três participantes do estudo atenderam ao critério e isso representa $<50 \%$; $\dagger$ Atributos não comparáveis; ¥Percentual de missings maior ou igual a 50\%.

Dentre os atributos comparáveis, encontram-se o acesso/acessibilidade, coordenação, integralidade dos serviços prestados e disponíveis, orientação comunitária e os escores geral, essencial e derivado. O PCAT-hanseníase é pautado no marco teórico da APS $^{(9)}$ e nas $\mathrm{ACH}$ preconizadas na diretriz nacional, a "orientação profissional" não é contemplada no PCAToolBrasil $^{(10)}$, mas, recomendada em outro estudo já que o SUS oferece capacitações ${ }^{(12)}$. Além disso, a "orientação familiar" e a "longitudinalidade" foram atributos não calculados para o PCAThanseníase, pois $80 \%$ dos participantes não acompanharam casos, critério para que 0 informante respondesse aos itens.
Constataram-se divergências na avaliação da APS no geral $(6,8)$ e na hanseníase $(6,1)$ o que evidencia dificuldades no desempenho das ações da doença quando comparadas às atividades realizadas de forma geral na APS. Fatores como profissionais despreparados, diminuição da incidência, estigma dos pacientes em tratar próximos às residências, dificuldade de acesso ao serviço e a resolutividade da APS são aspectos que dificultam a integração das $A C H$ na $A S^{(9)}$. Entretanto, forte orientação para APS no geral evidencia aproximação com os preceitos organizacionais e ideológicos do SUS; resultados semelhantes foram encontrados em Cascavel ${ }^{(13)}$, demonstrando o potencial da APS em assumir o protagonismo no desenvolvimento das $\mathrm{ACH}$. 
O acesso/acessibilidade teve percentual de baixa orientação em ambos os instrumentos, apesar disso, a média foi estatisticamente menor para a APS no geral. A acessibilidade é a utilização do serviço como fonte de cuidado a cada novo problema e o acesso compreende a questão geográfica, ou seja, é o modo como as pessoas chegam ao serviço ${ }^{(2)}$. Um estudo de revisão sistemática constatou inadequação no acesso/acessibilidade na maioria dos estudos que utilizaram o PCATool-Brasil ${ }^{(14)}$. Barreiras geográficas e organizacionais da APS, como reduzido horário de funcionamento, dificuldades para marcação de consultas e tempo de espera para atendimento contribuem para pior avaliação ${ }^{(14)}$. Esses obstáculos refletem-se na hanseníase, pois o usuário com suspeita da doença pode ter dificuldade, e assim retardar o diagnóstico precoce e o acompanhamento do caso. Em especial na hanseníase, o acesso é determinado pela priorização na política municipal, presença de profissionais capacitados, instrumentos que permitam avaliação dermatoneurológica e o conhecimento da população sobre a doença ${ }^{(15)}$.

Diferenças significativas foram observadas na integralidade dos serviços prestados e disponíveis; neste último, houve percentual de alto escore maior para as $\mathrm{ACH}$ quando comparado ao da APS no geral. A integralidade dos serviços prestados refere-se aos itens abordados pelo profissional entrevistado durante $\mathrm{o}$ atendimento ${ }^{(10)}$. Houve forte orientação em ambas as ferramentas e diferença significativa, porém as $\mathrm{ACH}$ pior avaliadas. Em relação ao PCAT-hanseníase, são ações preconizadas: a suspeição de casos, diagnóstico, orientações gerais, administração de dose supervisionada e avaliação dos contatos $^{(7)}$. No PCATool-Brasil a integralidade dos serviços prestados refere-se às políticas de promoção da saúde, segurança no ambiente domiciliar e orientações gerais quanto ao uso racional dos medicamentos ${ }^{(10)}$. Estudos realizados em Minas Gerais e no Pará, obtiveram, respectivamente forte e fraca orientação para as $\mathrm{ACH}^{(9,16)}$. Dessa maneira, diferentes orientações da APS nas $A C H$, sugerem que as desigualdades no desempenho se relacionam ao cenário epidemiológico, nível de integração das $\mathrm{ACH}$, aspectos políticos, culturais e socioeconômicos.

A integralidade dos serviços disponíveis são procedimentos e orientações executados na APS $^{(10)}$. No geral obteve-se escore limítrofe $(6,6)$, no entanto, no PCAT-hanseníase houve forte orientação $(8,9)$ e diferença estatística significativa. Itens do PCATool-Brasil podem ter contribuído para pior avaliação, pois questões como a execução de sutura, avaliação de problemas visuais, colocação de tala, remoção de verruga e unha encravada e inclusão em programa de suplementação alimentar ainda não são práticas rotineiras da APS. Em Lagoa Santa, como há apenas a ESF, em teoria apresenta características para efetivar os princípios da integralidade uma vez que as ações são realizadas no território, permitindo o planejamento de acordo com as necessidades e demandas a partir das condições de vida da população adscrita ${ }^{(1,17)}$.

Ao avaliar os atributos derivados, observase que a orientação comunitária apresentou, em ambos instrumentos, fragilidades na sua presença e extensão, contudo o grau de orientação foi estatisticamente menor para as $\mathrm{ACH}$. Destaca-se que a APS apresentou um comportamento heterogêneo nos escores essencial, derivado e geral, com diferenças significativas nos escores essencial e derivado.

Na orientação comunitária, constataram-se fragilidades na APS em geral e na hanseníase com diferença significativa. A orientação comunitária consiste no reconhecimento por parte do serviço das necessidades da comunidade por meio de indicadores epidemiológicos e do contato com a comunidade $^{(2)}$. Dificuldades em desempenhar as ações podem relacionar-se à persistência do modelo curativista baseado, principalmente, na doença, distante do modelo de vigilância em saúde. "A integração entre a Vigilância em Saúde e Atenção Básica é condição essencial para o alcance de resultados que atendam às necessidades de saúde da população, na ótica da integralidade da atenção à saúde" ${ }^{\prime 1)}$. Essas dificuldades refletem-se nas ACH pior avaliadas, pois o desconhecimento dos profissionais em relação à doença não se traduz em práticas que contemplem a comunidade e impactem nos problemas de saúde ${ }^{(16)}$.

$\mathrm{Na}$ coordenação, evidenciou-se forte orientação na APS em geral e na hanseníase, porém não houve diferença estatística. A coordenação é compreendida como o acesso aos serviços de forma integral em diferentes pontos da rede de atenção onde há interlocução entre os serviços $^{(2)}$. Dessa forma, para ter uma APS fortemente orientada para as $\mathrm{ACH}$ torna-se essencial que ela assuma o papel de ordenadora do cuidado e que serviços de média e alta complexidade funcionem, de preferência, 
mediante encaminhamentos de curta duração ${ }^{(9)} \mathrm{e}$ de acordo com os critérios definidos pela diretriz nacional ${ }^{(7)}$.

Salienta-se que houve avaliações isoladas de alguns atributos devido às questões conceituais: no PCATool-Brasil, os atributos logitudinalidade e orientação familiar, e no PCAThanseníase os atributos porta de entrada e orientação profissional.

No escore essencial, constatou-se fraca orientação no PCATool-Brasil, forte orientação no PCAT-hanseníase e diferença significativa. Na APS no geral, destaca-se que a integralidade dos serviços disponíveis e o acesso/acessibilidade contribuíram para pior avaliação, quando comparado com o PCAT-hanseníase. Quando o acesso/acessibilidade é facilitado, amplia-se a precocidade do diagnóstico e contribui na redução das incapacidades causadas pela doença ${ }^{(9)}$.

O atributo essencial "porta de entrada" (PCAT-hanseníase) obteve forte orientação e demonstra que a APS é compreendida como o local de escolha no caso de suspeição da doença, resultados semelhantes foram observados por enfermeiros ${ }^{(16)}$.

De maneira isolada, avaliação da "longitudinalidade" pela aplicação do PCAToolBrasil teve como resultado escore limítrofe $(6,9)$, porém uma APS fortemente orientada. Esse atributo é previsto na Política Nacional de Atenção Básica na qual estimular o vínculo tem como objetivo evitar a perda de referência, diminuir os riscos de iatrogenia decorrentes do desconhecimento e da coordenação do cuidado ${ }^{(1)}$ construída por fortes laços interpessoais que refletem cooperação mútua entre comunidade e profissionais ${ }^{(2)}$. Revisão sistemática realizada com estudos do PCATool-Brasil evidenciou que a longitudinalidade apresentou terceira melhor avaliação nos estudos ${ }^{(14)}$. Características como adscrição da população, universalização do acesso pela expansão da $\operatorname{ESF}^{(18)}$, organização do trabalho e equipe de referência ao longo do tempo ${ }^{(19)}$ podem ser variáveis que explicam o bom desempenho ${ }^{(14)}$.

Essas evidências corroboram a hipótese de que a APS é propícia para estabelecer o vínculo entre pacientes de hanseníase e equipes, pois a propedêutica da doença é longa, cerca de 6 a 12 meses, conforme classificação operacional da doença. Dessa forma, a integração das $\mathrm{ACH}$ na atenção primária é capaz de aumentar a detecção precoce, melhorar a adesão ao tratamento, prevenir as incapacidades e vigilância dos contatos por meio da vigilância em saúde ${ }^{(8)}$.

O escore derivado é conhecido por aumentar o poder de interação entre indivíduos e comunidade ${ }^{(10)}$. Constatou-se fraca orientação nas $\mathrm{ACH}(3,9)$, entretanto, forte orientação na APS no geral $(7,6)$ e diferença estatística significativa. A pior avaliação observada na hanseníase é retrato das fragilidades na orientação comunitária e profissional.

$\mathrm{Na}$ orientação profissional (PCAThanseníase), os resultados demonstraram fraca orientação da APS; $64,3 \%$ dos médicos/enfermeiros não realizaram treinamentos. Os treinamentos são importantes estratégias de fortalecimento da APS, uma vez que sensibilizam os profissionais pela situação epidemiológica. Além de capacitações, estratégias como busca ativa e realização de ações de educação em saúde são possibilidades para o diagnóstico precoce ${ }^{(20)}$. Vale mencionar que a falta de formação profissional em $\mathrm{ACH}$ junto a problemas no reconhecimento das necessidades da comunidade tem como consequência inadequada qualidade das $\mathrm{ACH}$, assim, a doença persiste como problema de saúde pública.

Outro atributo derivado, a orientação familiar (PCATool-Brasil), compreendida como avaliação das necessidades considerando o contexto familiar ${ }^{(2)}$ teve forte orientação da APS no geral, porém resultados diferentes foram observados em outros estudos que constataram pouca incorporação da família nas práticas assistenciais e desinteresse dos profissionais em conhecer as condições de vida do paciente e seus familiares ${ }^{(21-22)}$.

$\begin{array}{rlll}\text { Estudo realizado em } & \text { Tamandaré/ } \\ \text { Pernambuco constatou que } 93 \% \text { dos }\end{array}$
entrevistados mencionaram o tratamento como a ação prioritária na hanseníase seguido da consulta de enfermagem (86\%), prevenção de incapacidades $(79 \%)$ e controle clínico $(71 \%)^{(23)}$.

Isso demonstra inadequada inserção da família na propedêutica da doença que, constituída à imagem e semelhança da tecnociência e do modelo biomédico, ainda está incutida nas práticas de atenção à saúde dos profissionais ${ }^{(24)}$. Estudo que avaliou o conhecimento dos enfermeiros sobre o tratamento da hanseníase na APS evidenciou pouca ênfase da família como apoio nos casos da doença ${ }^{(25)}$. 
Tem-se como limitação que este estudo foi realizado em apenas um município, restringindo generalizações, assim, espera-se que estudos futuros sejam realizados com 0 intuito de aumentar o poder de comparabilidade entre as ferramentas de avaliação.

\section{CONCLUSÃO}

Em relação aos atributos comparáveis, constatou-se comportamento convergente na acessibilidade/acesso e orientação comunitária, inadequadamente avaliada, tanto no geral como nas $\mathrm{ACH}$. No escore essencial, houve divergência, pois a APS no geral foi pior avaliada do que na hanseníase. Entretanto, nos escores derivado e geral, constatou-se o inverso, uma vez que a APS no geral foi mais bem avaliada do que na hanseníase.

Conclui-se que o desempenho da APS no geral é melhor, isso demonstra as fragilidades na política municipal de saúde do município no enfrentamento da endemia. Assim, constata-se a importância de integrar as ações de hanseníase na APS, visto que, apesar de algumas fragilidades, o serviço é capaz de realizar as ações da doença.

A qualidade observada na APS pode refletir nas $\mathrm{ACH}$ por meio da priorização da doença no cenário epidemiológico, capacitação dos profissionais, conhecimento das necessidades, educação em saúde, envolvimento da gestão e do serviço de referência.

\section{REFERÊNCIAS}

1. Ministério da Saúde (BR). Departamento de Atenção Básica. Portaria MS/GM no 2436, de 22 de setembro de 2017. Aprova a Política Nacional de Atenção Básica, estabelecendo a revisão de diretrizes para a organização da Atenção Básica, no âmbito do Sistema Único de Saúde (SUS). Diário Oficial da União 2017.

2. Starfield B. Atenção primária: Equilíbrio entre necessidades de saúde, serviços e tecnologia. Brasília: UNESCO; 2002.

3. Vitória AM, Harzheim E, Takeda SP, Hauser L. Avaliação dos atributos da atenção primária à saúde em Chapecó, Brasil. Rev Bras Med Fam Comunidade 2013;8(29):285-93. DOI: 10.5712/rbmfc8(29)832

4. Silva SA, Nogueira DA, Paraizo CMS, Fracolli LA. Avaliação da Atenção Primária à Saúde: Visão dos profissionais de saúde. Rev Esc Enferm USP. 2014;48 (nesp):126-32. DOI: 10.1590/S0080$\underline{623420140000600018}$
5. Martins JS, Abreu SCC, Quevedo MP, Bourget MMM. Estudo comparativo entre Unidades de Saúde com e sem Estratégia Saúde da Família por meio do PCATool. Rev Bras Med Fam Comunidade 2016;11(38):1-13. DOI: 10.5712/rbmfc11(38)1252

6. World Health Organization (WHO). Global leprosy update, 2015: Time for action, accountability and inclusion. Geneva: World Health Organization; 2016.

7. Ministério da Saúde (BR). Secretaria de Vigilância em Saúde. Diretrizes para vigilância, atenção e eliminação da hanseníase como problema de Saúde Pública, com a finalidade de orientar os gestores e os profissionais dos serviços de saúde: Portaria MS/GM no 149, de 3 de fevereiro de 2016. Brasília, DF: Ministério da Saúde; 2016.

8. Saltarelli RMF, Seixas DHT. Limites e possibilidades na atenção ao portador de hanseníase no âmbito da estratégia saúde da família. Rev APS. 2016 [citado em 20 nov 2017]; 19(4):613-22. Disponível em: https://aps.ufif.emnuvens.com.br/aps/article/vie $\mathrm{w} / 2443$

9. Lanza FM. Avaliação da atenção primária no controle da hanseníase: Validação de instrumento e análise de desempenho de municípios endêmicos de Estado de Minas Gerais [tese]. Belo Horizonte (MG): Escola de Enfermagem da Universidade Federal de Minas Gerais; 2014.

10. Ministério da Saúde (BR). Secretaria de Atenção em Saúde. Manual do instrumento de avaliação da atenção primária à saúde: Primary care assessment tool PCATOOL. Brasília, DF: Ministério da Saúde; 2010.

11. Ministério da Saúde. Sala de Apoio a Gestão Estratégica. 2017 [citado em 12 dez 2017]; [1 tela]. Available in: http://sage.saude.gov.br

12. Organização Pan-Americana da Saúde. Validação de uma metodologia de avaliação rápida das características organizacionais e do desempenho dos serviços de atenção básica do Sistema Único de Saúde (SUS) em nível local. Brasília: Ministério da Saúde; 2006.

13. Carrer A, Toso BRGO, Guimarães ATB, Conterno JR, Minosso KC. Efetividade da Estratégia Saúde da Família em unidades com e sem Programa Mais Médicos em município no oeste do Paraná, Brasil. Ciênc Saúde Coletiva 2016;21(9):2849-60. DOI: 10.1590/141381232015219.16212016

14. Prates ML, Machado JC, Silva LS, Avelar PS, Prates LL, Mendonça ET, et al. Desempenho da 
Atenção Primária à Saúde segundo o instrumento PCATool: Uma revisão sistemática. Ciênc Saúde Coletiva 2017;22(6):1881-93. DOI: 10.1590/141381232017226.14282016

15. Lanza FM, Lana FCF. Decentralization of leprosy control actions in the micro-region of Almenara, State of Minas Gerais. Rev Latino-Am Enfermagem 2011;19(1):187-94. DOI: 10.1590/S0104-11692011000100025

16. Sousa GS, Silva RLF, Xavier MB. Atributos da atenção primária em saúde no controle da hanseníase: Ótica do enfermeiro. Rev Baiana Enferm. 2017;31(1):1-10. DOI: 10.18471/rbe.v31i1.17251

17. Sala A, Luppi CG, Simoes Z, Marsiglia RG. Integralidade e Atenção Primária à Saúde: Avaliação na perspectiva dos usuários de unidades de saúde do município de São Paulo. Saude Soc. 2011;20(4):948-60. DOI: 10.1590/S0104-12902011000400012

18. Lima EFA, Sousa Al, Primo CC, Leite FMC, Lima RCD, Maciel ELN. An assessment of primary care attributes from the perspective of female healthcare users. Rev Latino-Am Enfermagem 2015;23(3):553-9. DOI: 10.1590/01041169.0496 .2587

19. Pereira $M J B$, Abrahão-Curvo $P$, Fortuna $C M$, Coutinho SS, Queluz MC, Campos LVO, et al. Avaliação das características organizacionais e de desempenho de uma unidade de Atenção Básica à Saúde. Rev Gaúcha Enferm. 2011;32(1): 48-55. DOI: $10.1590 /$ S1983-14472011000100006

20. Stafin I, Guedes VR, Mendes SUR. Diagnóstico precoce de Hanseníase e ações estratégicas para a sua detecção. Revista de Patologia do Tocantins 2018;5(2):67-73. DOI: 10.20873/uft.2446-6492.2018v5n2p67

21. Alencar MN, Coimbra LC, Morais APP, Silva AAM, Pinheiro SRA, Queiroz RCS. Avaliação do enfoque familiar e orientação para a comunidade na Estratégia Saúde da Família. Cien Saude Coletiva 2014;19(2):353-64. DOI: 10.1590/141381232014192.08522012

22. Silva NC, Giovanella L, Mainbourg EMT. A família nas práticas das equipes de Saúde da família. Ver Bras Enferm. 2014;67(2):274-81. DOI: 10.5935/0034-7167.20140037

23. Silva LSR, Silva TM, Rocha JT, Andrade WG, Lessa EC, Correia NS. A assistência de enfermagem aos portadores de hanseníase assistidos pelo programa de saúde da família. RUOL. 2016 [citado em 3 mar 2018]; 10(11):411117.

Disponível https://periodicos.ufpe.br/revistas/revistaenferm agem/article/view/11498/13365

24. Santos SSBS, Silva LS, Carneiro EKN, Saback MAMC, Carvalho ESS. Processo de trabalho da equipe de Enfermagem em Unidades Saúde da Família em município baiano. Rev Baiana Enferm. 2013;27(2):101-7.

DOI:

http://dx.doi.org/10.18471/rbe.v27i2.8850

25. Ribeiro MDA, Castillo IS, Silva JCA, Oliveira $S B$. The nurse's view on leprosy treatment in primary health care. Rev Bras Promoc Saúde 2017 [citado em 16 jul 2018]; 30(2):221-8. Disponível em:https://search.proquest.com/openview/fb07 9e9f004efd $1 \mathrm{fdcf} 7195 \mathrm{fc} 543579 \mathrm{c} / 1$ ? pqorigsite $=\mathrm{gsc}$ holar\&cbl=2046042

Nota: Artigo elaborado a partir dos Relatórios técnicos finais dos projetos Vigilância, prevenção e controle da hanseníase em Minas Gerais e Vigilância, prevenção e controle da hanseníase em Minas Gerais: distribuição espacial dos casos, avaliação dos serviços em saúde e análise da infectividade em contatos domiciliares. Projeto financiado com recursos da Fundação de Amparo à Pesquisa do Estado de Minas Gerais - FAPEMIG - Edital № 03/2014 - e da Secretaria de Vigilância em Saúde / Ministério da Saúde - SVS/MS - Edital 197/2012.

Recebido em: 22/04/2018

Aprovado em: 20/11/2018

Endereço de correspondência:

Nayara Figueiredo Vieira

Rua Zaida Torres, № 101, apt302

CEP: 38610-000 - Unaí/MG - Brasil

E-mail: nayarafv5@hotmail.com 